\title{
Structure of salts solution in polar dielectric liquids and electrically induced separation of solvated ions
}

\author{
Igor V. Shamanin ${ }^{\mathrm{a}}$, Mishik A. Kazaryan ${ }^{\mathrm{b}}$, Victor I. Sachkov ${ }^{\mathrm{c}}$ \\ ${ }^{a}$ National Research Tomsk Polytechnic University, Technical Physics Department, Lenina Prosp., \\ 30, Tomsk, Russia; ${ }^{b}$ Physical Institute of Russian Academy of Sciences, Leninskiy Prosp., 53, \\ Moscow, Russia; 'Siberian Physical-Technical Institute of Tomsk \\ State University, 1 Novosobornaya sq., Tomsk, Russia
}

The aim of study is to demonstrate that separation of solvated ions in solution of mix of salts under the action of external periodic electric field happens because of around ions there are formed clusters consisting of molecules of solvent and the sizes of such clusters have dimensions $\sim 0.1 \mu \mathrm{m}$.

In investigations the sizes of clusters theoretically were defined and experimentally value of frequency of external electric field which action excites the effect of separation of the solvated ions was defined.

Experiments were done in the Technical Physics Chair of the National Research Tomsk Polytechnic University.

At theoretical determination of the dimensions of clusters Poisson's equation was solved and was considered that polar molecules of solvent are oriented under the action of electric field of an ion. The chemical composition of samples of solutions was determined by means of the spectrophotometry and he X-ray excited fluorescent radiation analysis method.

Theoretical estimates and results of experiments confirmed the assumption that clusters which are formed around ions in solutions have the dimensions $\sim 0.1 \mu \mathrm{m}$.

Results of investigation testify that placing of volume distributed electric charge of ion in dielectric liquid is accompanied by formation of the supramolecular particles, which we called "clusters", linear sizes of which is significantly more than first and second radiuses of solvation ( $\sim 1$ Angstrom $)$ and reach size $\sim 0.1 \mu \mathrm{m}$. At such sizes inertial properties of clusters and their natural frequencies give the chance to operate their movement by means of action of external electric field on solution.

Keywords: Solution, cation, solvation, electric field, directed motion, separation.

\section{INTRODUCTION}

In experiments when external periodic electric field acts to solution of mix of salts in water the effect of separation of the hydrated cations was found. Thus electrodes which form electric field were electrically isolated from solution. Potential of one of electrodes changed in time as a sine and with different amplitudes in half-cycles. We called such electric field asymmetric and the ratio of values of amplitudes - asymmetry coefficient. Results of research of this effect are presented in the monograph [1]. When the frequency of change of potential (frequency of an external field) was $\sim 100 \mathrm{~Hz}$ up to 2 $\mathrm{kHz}$ separation of the solvated cations in solution was observed. At increase in frequency the effect disappeared.

It was natural to assume that the frequency of external electric field has to correlate with natural frequency of particles moving of which is directed in volume of solution under the action of this field. These particles are formed by cations and solvent molecules which are associated around cations. Such particles are called as solvated cations. According to existing point of view the sizes the solvated cations don't exceed 10 Angstrom and no more than 2 layers which are formed by solvent molecules around a cation are taking in account. Estimates showed that value of natural frequency of solvated cations oscillations is $\sim 10 \mathrm{MHz}$ and more. But we found effect with significantly smaller frequencies. It is meant that the size and mass of a particle which consists of a cation and solvent molecules around it must be significantly more than 10 Angstrom. It is possible to assume that under certain conditions in solution when solvent is polar dielectric (for example water) around ions in the connected state there is large number $\left(\sim 10^{5}\right)$ of molecules of solvent.

International Conference on Atomic and Molecular Pulsed Lasers XII, edited by Victor F. Tarasenko,

Andrey M. Kabanov, Proc. of SPIE Vol. 9810, 981019 · (c) 2015 SPIE

CCC code: $0277-786 \mathrm{X} / 15 / \$ 18 \cdot$ doi: $10.1117 / 12.2225223$ 
The purpose of our investigation is to determine the sizes of particles which are formed in salt solution in polar dielectric liquid and check the opportunity to use the founded effect for separation of cations of different metals.

\section{FORMATION OF CLUSTERS IN SOLUTIONS OF SALTS IN POLAR DIELECTRIC LIQUIDS}

Condition and properties of molecules of solvent of both types - cations and anions - which are under action of electric fields in solution are described by identical laws. As changes of cations concentration in solution was measured in experiments, subjects of the description in the paper are cations.

\subsection{Cation's Electric Field Distribution in Solution}

Solvent is dielectric liquid. Molecules of solvent are the polar molecules (for example, water). Distribution of density of polarizing charge induced by an ion in volume of solvent is defined by the relation:

$$
\rho_{\text {polar }}=-\nabla \vec{P}
$$

in which the vector of polarization $\vec{P}$ is linearly related with the electric field strength $\vec{E}$ which is created by the ion:

$$
\vec{P}=\chi \varepsilon_{0} \vec{E}
$$

where $\chi$ is the dielectric susceptibility of the solvent and $\varepsilon_{0}$ is the electric constant.

Ion having the charge of $q$ creates the field distribution of which in the dielectric solvent is described by the relation:

$$
\vec{E}=\frac{q \vec{r}}{4 \pi \varepsilon_{0} \aleph r^{3}},
$$

where $\aleph=1+\chi$ is the dielectric permeability of solvent, $\vec{r}$ is the radius-vector with origin at the ion geometrical center.

The relation (1) is mathematical model of physical process of formation of a resultant charge in the dielectric caused by non-uniform polarization.

Taking into account that the radial component of the electric field strength vector $E_{r}=\frac{q}{4 \pi \varepsilon_{0} \aleph r^{2}}$, and the field of the central ion is spherically symmetric, it is easy to obtain the relation:

$$
\rho_{\text {polar }}=\chi \frac{q}{2 \pi \aleph} \frac{1}{r^{3}} .
$$

Thus, density of the induced (polarizing) charge is inversely proportional to the third degree of the distance counted from the center of an ion.

When electric field is absent, molecules are randomly oriented in various directions. Therefore the total dipolar moment in unit of volume is equal to zero.

In the electric field of a central ion (cation or anion) at once there are two processes: first, the additional dipolar moment because of forces acting on electrons (electric polarizability) is induced; secondly, electric field seeks to focus (to direct) each of molecules, creating resultant, non- zero moment in unit of volume. Collision of molecules at their chaotic 
Brownian motion doesn't allow them to keep strictly fixed orientation, but formation of the ordered structure around an ion nevertheless is possible. We called this structure as "cluster".

In the first approximation the molecule of dielectric solvent can be considered as the dipole having the dipolar electric moment:

$$
\overrightarrow{p_{0}}=Q \vec{l}
$$

where $Q$ is the absolute value of the total positive and total negative charges located, respectively, in the centers of these charges; $\vec{l}$ is the distance between centers of positive and negative charges.

Such dielectric is called as polar dielectric, molecules (atoms) of which have the electrons located asymmetrically relatively to the nuclei of constituting atoms (for example $\mathrm{H}_{2} \mathrm{O}, \mathrm{HCl}, \mathrm{NH}_{3}, \mathrm{CH}_{3} \mathrm{Cl}$, etc.). In such molecules the centers of positive and negative charges don't coincide, being, practically, at constant distance from each other. Molecules of polar dielectrics on their electric properties are similar to rigid dipoles which have a constant dipolar moment $\overrightarrow{p_{0}}=$ const . The rigid dipole placed in a uniform external electrostatic field, is under action of couple of forces with the moment equal to:

$$
\vec{M}=\left[\overrightarrow{p_{0}} \vec{E}\right]
$$

The moment of couple of forces $\vec{M}$ is directed perpendicularly to the plane passing through vectors $\overrightarrow{p_{0}}$ and $\vec{E}$, and from the end of vector $\vec{M}$ rotation from $\overrightarrow{p_{0}}$ to $\vec{E}$ must happens on the shortest way, passing counterclockwise.

In real molecules of polar dielectrics besides the turn of axes of dipoles along a field, it takes place the deformation of molecules, and in them some induced dipolar moment is created.

If polar dielectric isn't placed in external electric field, as a result of chaotic thermal motion of molecules vectors of their dipolar moments are directed chaotically. Therefore the sum of the dipolar moments of all molecules is equal to zero in any physically infinitesimal volume $\Delta V$. (Thus $\Delta V>>\mathrm{v}_{0}$, where $\mathrm{v}_{0}$ is the volume of one molecule, and volume $\Delta V$ contains very large number of molecules).

When entering dielectric into external electric field there is the dielectric polarization, consisting that in any elementary volume $\Delta V$ there is a total dipolar moment of molecules, other than zero. Dielectric which is in such state is called the polarized dielectric. In polar dielectrics there is the orientation polarization. Thus external electric field seeks to direct the dipolar moments of rigid dipoles along the direction of electric field. It is interfered by the chaotic thermal motion of the molecules seeking "to scatter" dipoles randomly. As a result of joint action of a field and thermal movement there is the preferred orientation of the dipolar electric moments along the field, increasing with increase in the strength of electric field and reduction in temperature.

Quantitative measure of polarization of dielectric is the polarization vector $\vec{P}$. As vector of polarization is called the ratio of the electric dipolar moment of small volume $\Delta V$ of dielectric to this volume:

$$
\vec{P}=\frac{1}{\Delta V} \sum_{i=1}^{N} \overrightarrow{p_{i}}
$$

where $\overrightarrow{p_{i}}$ is the electric dipolar moment of $i$-th molecule; $N$ is the total number of molecules in volume. 
This volume has to be so small that in it electric field could be considered uniform. At the same time the number of molecules $N$ in volume $\Delta V$ has to be rather great in order that it was possible to apply statistical methods of description.

For the uniform polar dielectric which is placed in uniform electric field:

$$
\vec{P}=\bar{n}<\overrightarrow{p_{0}}>
$$

where $\left\langle\vec{p}_{0}\right\rangle$ is the average value of a component of the constant dipolar moment of a molecule along direction of the field.

If polar dielectric is placed in the external electric field of small strength, the dielectric susceptibility may be calculated with use of Langevin-Debye formula:

$$
\chi=\frac{\bar{n} p_{0}^{2}}{3 \varepsilon_{0} k T}
$$

Taking into account that $\chi=\aleph-1$, it is easy to obtain the equation:

$$
\rho_{\text {polar }}=\frac{\bar{n} p_{0}^{2}}{3 \varepsilon_{0} k T} \frac{q}{2 \pi \aleph} \frac{1}{r^{3}},
$$

and Poisson's equation may be written in the form:

$$
\nabla^{2} \psi=-\frac{2}{3} \frac{\bar{n} p_{0}^{2} q}{\left(\varepsilon_{0} \aleph\right)^{2} k T} \frac{1}{r^{3}} .
$$

The equation (11) in spherical geometry may be written in the form:

$$
\frac{d^{2} \psi}{d r^{2}}+\frac{2}{r} \frac{d \psi}{d r}=\frac{a}{r^{3}},
$$

where $a=-\frac{2}{3} \frac{\bar{n} p_{0}^{2} q}{\left(\varepsilon_{0} \aleph\right)^{2} k T}$.

Boundary conditions for Poisson's equation in relation to a considered case will be written in the form:

$$
\psi\left(r=r_{0}\right)=\psi_{0} ; \psi(r \rightarrow \infty)=0,
$$

where $r_{0}$ is the ionic radius, $\psi_{0}$ is the ion's potential. Equality to zero of potential on infinite from a point charge follows from basic principles of electrostatics and electrodynamics of continuous matter. Potential of an ion decides as the potential of uniformly charged sphere ( $q$ is the value of charge) with radius $r_{0}$ [2]:

$$
\psi_{0}=\frac{1}{4 \pi \varepsilon_{0}} \frac{q}{r_{0}} .
$$

For $\mathrm{Y}^{3+}$ cation $q=4.8 \cdot 10^{-19} \mathrm{C}, r_{0}=1.06 \cdot 10^{-10} \mathrm{~m}, \varepsilon_{0}=8.854 \cdot 10^{-12} \mathrm{C} / \mathrm{V} \cdot \mathrm{m}$. Thus for $\mathrm{Y}^{3+}$ cation $\psi_{0}=40.699 \mathrm{~V}$. 
The equation (12) is the linear non-uniform equation of the 2-nd order with variable coefficients. Its solution may be written in the form:

$$
\psi(r)=-\frac{1}{r}\left(\ln \frac{C_{1}}{r^{a}}-a\right)+C_{2},
$$

where $C_{1}$ and $C_{2}$ are the constants determined by boundary conditions (13). It is clear that according to the second boundary condition $C_{2}=0$, and use of the first boundary condition gives the possibility to define:

$$
C_{1}=r_{0}^{a} \exp \left(r_{0} \psi\left(r_{0}\right)+a\right)
$$

For example, $C_{1}$ is 1 for cations $Y^{3+}$ and $C e^{3+}$. Value of constant $a$ is $-1.951 \cdot 10^{-10}$ for $T=298 \mathrm{~K}$ and $q=3|\bar{e}|$, where $\bar{e}$ is the electron charge.

Strength of electric field as function of argument $r$, in spherical system of coordinates, in case of the central symmetry is defined by the relation:

$$
E_{r}(r)=-\frac{d \psi}{d r}=-\frac{1}{r^{2}} \ln \frac{C_{1}}{r^{a}} .
$$

\subsection{Solvent Molecules Held by Cation's Electric Field}

Theoretical and experimental studies of processes of functional nanostructures formed in liquids [3] showed that due to a dipole-dipole interaction of molecules of dielectric liquids (in particular, water) formation of molecular "bridges" in the

liquid which is placed in electric field between electrodes is possible. Thus there is a critical electric field $E_{c r}$ for formation of molecular "bridges":

$$
E_{c r}=\frac{1}{\alpha}\left[\left(p_{0}^{2}+2 \alpha k T\right)^{1 / 2}-p_{0}\right],
$$

where $\alpha$ is the polarizability of a molecule of liquid.

On the Fig. 1 the function $E_{r}(r)$ in limits $0.1 \mu m \leq r \leq 0.75 \mu m$ for cations $\mathrm{Li}^{+}, \mathrm{Cd}^{2+}, \mathrm{Y}^{3+}$ placed in water is presented. 


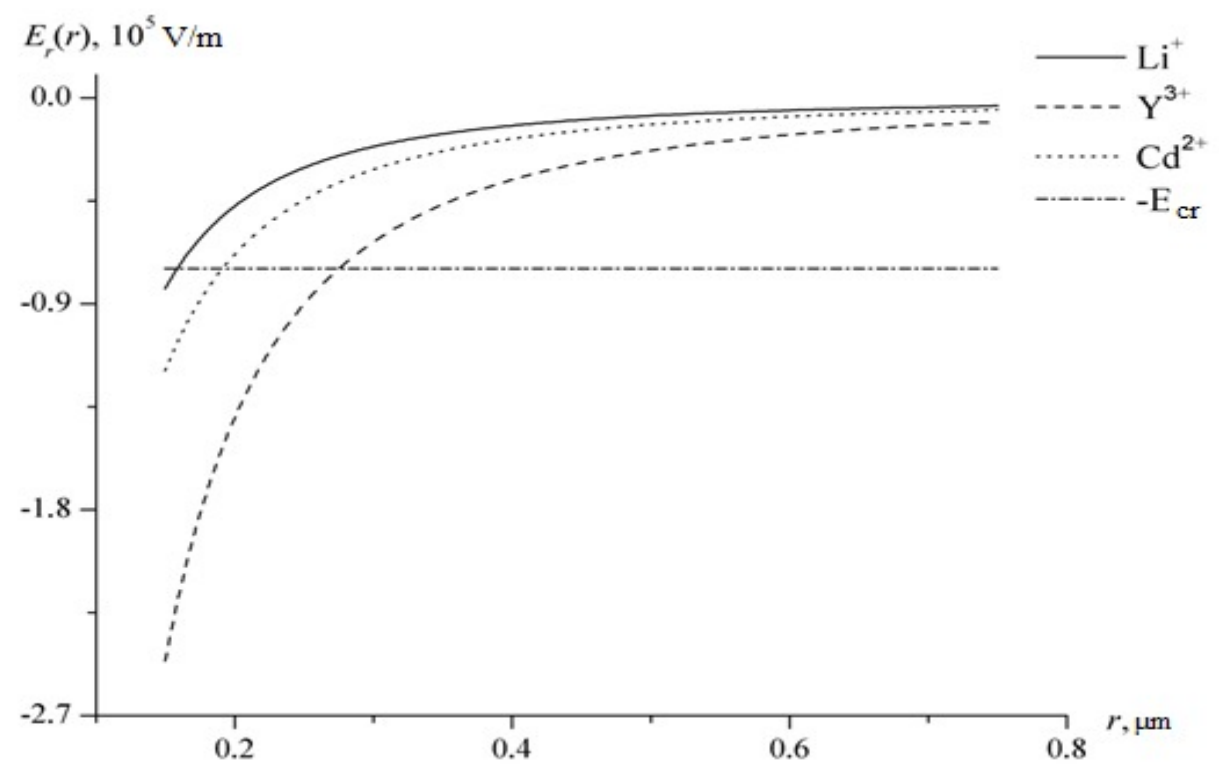

Fig. 1. Radial dependence of the electric field strength for $\mathrm{Li}^{+}, \mathrm{Cd}^{2+}, \mathrm{Y}^{3+}$ cations at temperature of $298 \mathrm{~K}$

At $|E|>\left|E_{\kappa p}\right|$ polarized molecules of solvent will be connected by dipole-dipole interaction and directed along the field of the central ion. At $|E|<\left|E_{\kappa p}\right|$ thermal motion of molecules of solvent has to destroy "molecular bridges". It is possible to assume that around the central ion the large number of molecular "bridges" is built, and each of "bridges" is a chain of the molecules of solvent built along electric field if ion. These "bridges" form the coating of the ion shielding its field. The cluster consists of the central ion and the polarized molecules of solvent surrounding ion are formed. Radius of such cluster $r_{c l}$ can be estimated from a condition $|E|=\left|E_{c r}\right|$ that is from the relation:

$$
\frac{1}{r_{c l}^{2}} \ln \frac{C_{1}}{r_{c l}^{a}} \approx \frac{1}{\alpha}\left[\left(p_{0}^{2}+2 \alpha k T\right)^{1 / 2}-p_{0}\right] \text {. }
$$

This condition means that at $r=r_{c l}$ the central ion field is compensated by the field of "bridges" of the built dipoles. That is, coating consisting of the built dipoles shields the central ion. The solution of the equation (19) gives values of $r_{c l}$ which are presented in Table 1.

Table 1. Sizes of clusters for ions of $\mathrm{Li}^{+}, \mathrm{Mg}^{2+}, \mathrm{Ca}^{2+}, \mathrm{Cd}^{2+}, \mathrm{Y}^{3+}$, being in water, at temperature of $298 \mathrm{~K}$

\begin{tabular}{|l|l|}
\hline Cation & Cluster radius, $\mu \mathrm{m}$ \\
\hline $\mathrm{Li}^{+}$ & 0.115 \\
\hline $\mathrm{Mg}^{2+}$ & 0.119 \\
\hline $\mathrm{Ca}^{2+}$ & 0,128 \\
\hline $\mathrm{Cd}^{2+}$ & 0.162 \\
\hline $\mathrm{Y}^{3+}$ & 0.275 \\
\hline
\end{tabular}


At such sizes of clusters value of own frequency of their oscillations $\sim 1 \mathrm{kHz}$. It is possible to assume that exactly due to formation of such clusters action of external electric field on solution excites directed motion of cations. As cations of different atoms have different inertial properties that parameters of directed motion of different cations in solution will differ.

\subsection{The Possibility of Solvated Ions Associates Formation}

The cluster radius value obtained in the approximation of self-existent field existence in the bulk solution leads to the evident question - "How does it relate to the characteristic value of the distance between the ions equally distributed in the bulk solution?"

Uncomplicated estimations show that in case of aqueous solution of hydrated yttrium nitrate $\mathrm{Y}\left(\mathrm{NO}_{3}\right)_{3} \cdot 6 \mathrm{H}_{2} \mathrm{O}$ at salt concentration of $10 \mathrm{~g} / \mathrm{l}$ the average value of the distance between equally distributed ions in the bulk solution is about $100 \mathrm{~nm}(0.1 \mu \mathrm{m})$. This value is considerably smaller than the evaluated values of the solvation shell radii (clusters radii) presented in table 1.

There is no contradiction in this fact. It point is that in the approximation of self-consistent filed existence the value of the screening constant is determined, while its reciprocal value determines the radius of the ion action field within the limits of which the solvent molecules "react" to this field action. To be more precise, the cluster radius can be called the radius of a single ion action and it can be meant that other ions can be situated within this radius. At high values of the salt concentrations the actions of separate ions radii overlap, as the cluster if formed by several ions having "common" solvation shell.

It is possible to assess the number of ions within the limits of such common solvation shell. For this purpose electrostatic energy of the cluster-associate formed by the controlled amount of solvated ions should be determined considering that columbic energy of co-ions (repulsive forces) is compensated by interaction energy (attraction forces) of the solvent molecules, which are situated within the limits of the "common" solvation shell.

Let us consider the associate as the roll radius $r_{c l}$, in the volume of which $n_{i}$ of the ions, for example, cations, are distributed. If the electric interaction energy of all ionic pairs is calculated, considering them (at first) as points equally distributed by the roll volume, it is equal to:

$$
U_{1}=\frac{3}{5} \cdot \frac{n_{i} \cdot\left(n_{i}-1\right)}{4 \pi \varepsilon_{0} \cdot r_{c l}} \cdot\left(z_{i} e\right)^{2}
$$

where $z_{i}$ - ion charge ratio, $e$ - electron charge.

Let us consider a separate ion as the roll radius $r_{i}$. The molecule of the solvent, which is a polar dielectric, is oriented by centrally symmetric ion field. The molecule is a dipole the energy if which in the field is equal to:

$$
U=-p_{0} \cdot E,
$$

where $p_{0}$ - is a dipolar moment of the solvent molecule: $p_{0}=\alpha \cdot \varepsilon_{0} \cdot E$, where $\alpha$ - molecular polarization of the solvent.

Molecules of the polar solvent orient within the sphere the radius $r_{*}$ of which is determined by the equation (19). Electric field intensity within this sphere limits was determined by the ratio (17).

We can get that the energy of interaction (attraction) of one solvent molecule with an ion is:

$$
U_{s i}(r)=-\alpha \cdot \varepsilon_{0} \cdot E(r)^{2}=-\alpha \cdot \varepsilon_{0} \cdot \frac{1}{r^{4}}\left[\ln \frac{C_{1}}{r^{a}}\right]^{2}
$$


The solvent molecules number in the solvation shell is approximately $N_{s} \approx\left(\frac{r_{*}}{r_{s}}\right)^{3}$. The energy of attraction of all solvent molecules oriented in the field and "affixed" to the ion

$$
U_{s} \approx-81 \cdot \frac{\alpha \varepsilon_{0}}{r_{*} \cdot r_{s}^{3}}\left[\ln \frac{3^{a} \cdot C_{1}}{r^{a}}\right]^{2}
$$

The associate contains $n_{i}$ ions, thus the total attraction energy of the solvent molecules, oriented in the field of all ions forming the associate, is

$$
U_{2} \approx n_{i} \cdot U_{S}
$$

The energy of dipole-dipole interaction between two molecules of the polar solvent for the case of collinear parallel arrangement of dipoles

$$
U_{d-d} \approx-\frac{2 \cdot \mu^{2}}{4 \pi \varepsilon_{0} d^{3}}
$$

where $\mu$-constant dipole moment of the solvent molecule $d$ - solvent molecule diameter, more precisely, the diameter of the sphere within the limits of which the entire separate solvent molecule can be situated. The energy of dipole-dipole interaction of all solvent molecular pairs of the associate and not influenced by the electric fields of separate ions

$$
U_{3} \approx-\left(\frac{6}{5}\right)^{3} \cdot \frac{n_{s} \cdot\left(n_{s}-1\right)}{8 \pi \varepsilon_{0} \cdot r_{c l}^{3}} \cdot \mu^{2}
$$

where $n_{s} \approx \frac{r_{c l}^{3}-n_{i} \cdot r_{*}^{3}}{r_{s}^{3}}$ - the number of solvent molecules, which are in the associate and are not oriented by separate ions fields. Determining $U_{3}$ it was taken into account that the average value $\left(\frac{1}{r_{i j}}\right)$ by all pairs of the points inside the roll radius $r_{c l}$ equals to $\frac{6}{\left(5 \cdot r_{c l}\right)}$.

Total electrostatic energy of the associate in the first approximation is determined as the sum

$$
U \approx U_{1}+U_{2}+U_{3} .
$$

It can be supposed that the minimum of the value $U$ should correspond to the optimum configuration of the associate, formed by $n_{i}$ ions, which are situated within the limits of roll radius $r_{c l}$. The general solvation shell of all ions is formed by the solvent molecules within the radius $r_{c l}$. The minimum of the values $U$ is determined by a simple differentiation of the total ration for $U$ either for the fixed value $r_{c l}$, or for the fixed value $n_{i}$.

Solving the equation $\frac{d U}{d n_{i}}=0$ at the given value of $r_{c l}$ allows determining the value $n_{i}$, corresponding to the minimum of associate electrostatic energy, while the solution of the equation $\frac{d U}{d r_{c l}}=0$ at the given $n_{i}$ allows 
determining the value $r_{c l}$, corresponding to the minimum of electrostatic energy. Organization of a rather simple iteration process allows determining the optimum combination of the values $n_{i}$ and $r_{c l}$, which at first approximation should correspond to the optimum associate configuration.

Solution to the equation $\frac{d U}{d n_{i}}=0$ has two forms:

$$
n_{i} \approx \frac{b+c+d-2 d\left(\frac{r_{c l}}{r_{s}}\right)^{3}}{2 b-2 d\left(\frac{r_{*}}{r_{s}}\right)^{3}},
$$

This allows determining the optimum number of cations forming the associate with the radius $r_{c l}$. The constants included into the last ratio are determined by the formulae:

$$
\begin{gathered}
b=\frac{3}{5} \cdot \frac{\left(z_{i} \cdot e\right)^{2}}{4 \pi \varepsilon_{0} \cdot r_{c l}} ; \\
c=81 \cdot \frac{\alpha \varepsilon_{0}}{r_{*} r_{s}^{3}} \cdot\left[\ln \frac{3^{a} C_{1}}{r_{s}^{a}}\right]^{2} ; \\
d=\frac{27}{125} \cdot\left(\frac{r_{*}}{r_{s}}\right)^{3} \cdot \frac{\mu^{2}}{4 \pi \varepsilon_{0} \cdot r_{c l}^{3}} .
\end{gathered}
$$

In case of aqueous solution of cerium at salt concentrations the values of constants are: $b \approx 10^{-22}, c \approx 5 \cdot 10^{-20}, d \approx 3 \cdot 10^{-25}$. For the cluster (associate) the radius of $20 \mu \mathrm{m}$ the assessment carried out by the ratio (28) gives the value of $n_{i} \approx 10^{5}$.

\section{EXPERIMENTAL RESULTS (WITHOUT SOLUTION CIRCULATION)}

To carry out the experiment for studying the process of selective drifting the aqueous solution of $\mathrm{Ce}\left(\mathrm{NO}_{3}\right)_{3}$ and $\mathrm{Pb}\left(\mathrm{NO}_{3}\right)_{2}$ salts mixtures with the metal concentration of $0.1 \mathrm{~g} / \mathrm{l}$ was used. Spectrophotometry method was used for quantitative estimation of the solution concentration change. This method allows estimating the content of element by the line absorption intensity in a characteristic spectrum.

The selected samples analysis was carried out using the spectrophotometer EVOLUTION UV600 with the spectral range in ultra-violet region. The spectrophotometer is for measuring spectral transmittance of liquids and solid materials in the spectrum range from 190 to $1100 \mathrm{~nm}$, as well as for measuring coefficients of scattered and direct reflection of plane objects. The error of the wavelength setting is not more than $0.03 \mathrm{~nm}$; the playback accuracy error is not more than \pm 0.1 $\mathrm{nm}$; photometric range: transmission optical density (A): $-0.3-4$ A ; researches speed $0.05 ; 0.1 ; 0.2 ; 1 ; 2 ; 5$ and $10 \mathrm{~nm} / \mathrm{s}$.

Using the UV-spectrometer the solutions samples with the following parameters were studied after experiments:

Ce concentration, $g / 1$

$\mathrm{Pb}$ concentration, $\mathrm{g} / \mathrm{l}$

Electric field frequency, $\mathrm{Hz}$

Ambient temperature, ${ }^{\circ} \mathrm{C}$

Pressure, Mhdg
0.1

0.1

100 and 200

22

$747-752$ 
Amplitude of the first positive half-wavy, V/sm Electric signal asymmetry coefficient Electric field operation time, $\mathrm{h}$
78.9-98.7

$0.2 ; 0.5$

$215 ; 430,8,20$

The obtained spectra were read and processed using the program VISIONpro. According to the curves for solutions with the identified concentrations the calibration schedule was built, on the basis of which cerium cations concentrations were determined.

Experimental installation consists of three-section cell (fig. 2). The cell dimensional specifications: length: width: depth: $-10: 10: 10 \mathrm{~cm}$. Useful (internal) volume $-320 \mathrm{ml}$. Three cell sections: $\mathrm{s} 1-\mathrm{s} 3$ (fig. 2, pos. 2). Potential of one of electrodes of separation cell changed in time as sine, but with different amplitudes in half-cycles

$$
U(t) \approx A(1+2 \sin \omega t-\sin 2 \omega t) .
$$

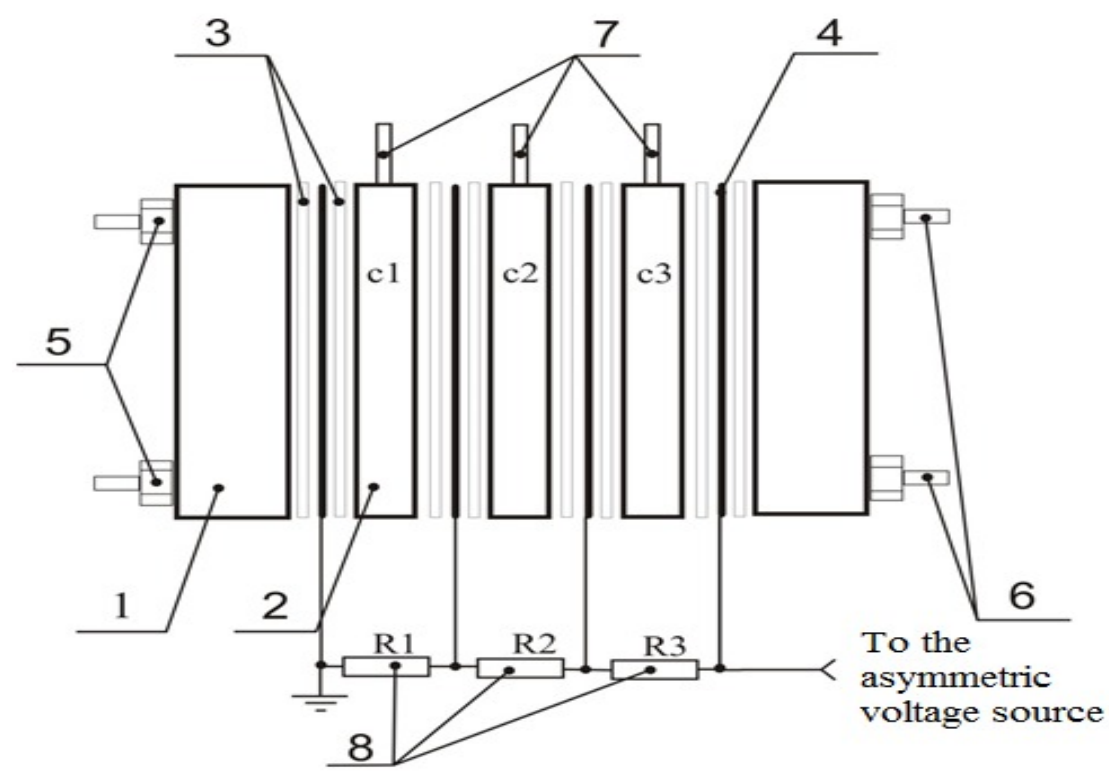

Fig. 2. Experimental cell

The samples were selected after 2 hours 15 minutes; 4hours 30 minutes, 8hours and 20 hours of high frequency asymmetric field influence on aqueous solutions of $\mathrm{Ce}\left(\mathrm{NO}_{3}\right)_{3}$ and $\mathrm{Pb}\left(\mathrm{NO}_{3}\right)_{2}$.

Previously it was shown in numerical experiments [1], that when the solution of salt was influenced by electric field with equal stress amplitudes in positive and negative half-periods ("symmetric field"), selective drifting of solvated ions was not excited. Selective drifting effect should be expected only in case of asymmetric electric signal.

Experimental results for the case with "symmetric field" are shown in fig. 3. 


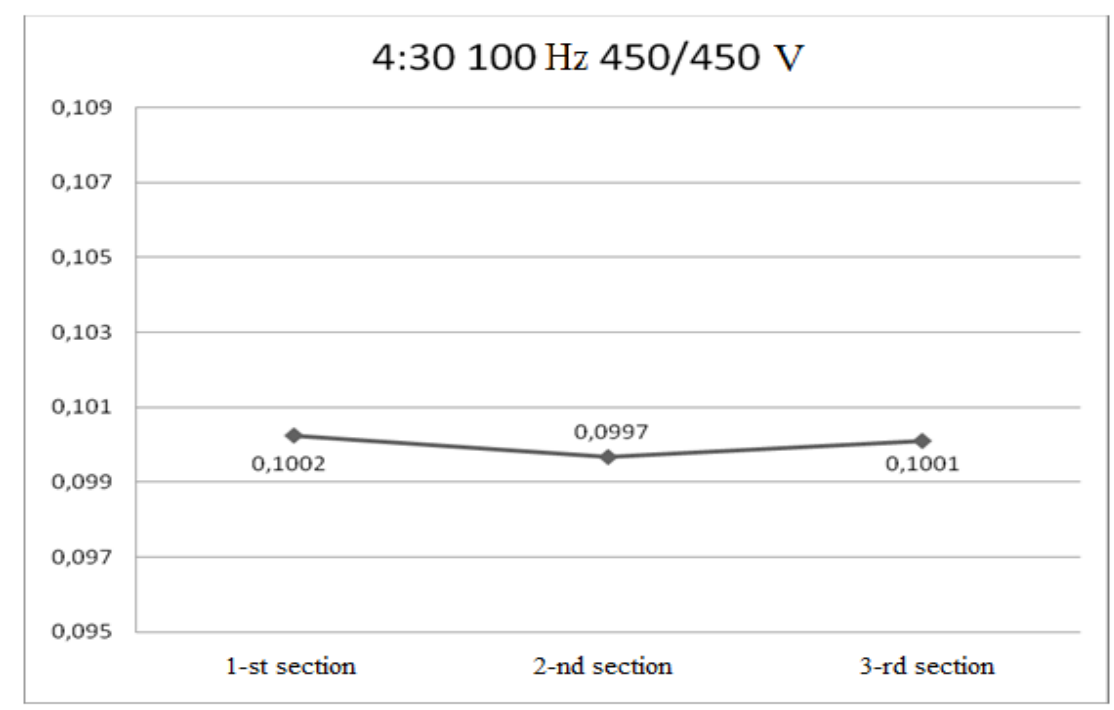

Fig. 3. Cerium cations concentration $(\mathrm{g} / \mathrm{l})$ in the experimental cell after field action for 4 hours $30 \mathrm{minutes}$. $100 \mathrm{~Hz}$, $450 / 450 \mathrm{~V}$.

When "symmetric field" acts on the solution, that is the coefficient of electric signal asymmetry is equal to 1 , the concentrations changes in sections did not exceed $0.4 \%$ (fig. 3). Such insignificant changes can be explained by the measurement error.

When the solution is influence by the field with the frequency of $200 \mathrm{~Hz}$, asymmetry coefficient of 0.5 and positive halfperiod amplitude of $600 \mathrm{~V}$, there is a tendency of solvated cerium cations concentrations increase in sections 1 and 3 , the solution depletes in 2-nd section. The separation coefficient (at selection in 1-st section is 1.015. When the field acts for 4 hours 30 minutes the tendency is unstable.

When the frequency decreases to $100 \mathrm{~Hz}$, other field parameters remaining the same, the tendency for the grounded electrode to increase concentration becomes more defined. In 1-st section there is marked increase of cerium cations concentration, a slightly smaller increase is detected in 2-nd section, while the solution is depleted by solvated cations in 3-rd section. The electric field action for 4 hours 30 minutes is close to optimum, as the separation coefficient (at selection in 1-st section is 1.054 at that moment (fig. 4). When the time increases to 8 hours, the separation coefficient decreases to 1.026 . The decrease of the field action to 2 hours 30 minutes also decreases the separation coefficient to 1.0166 . 


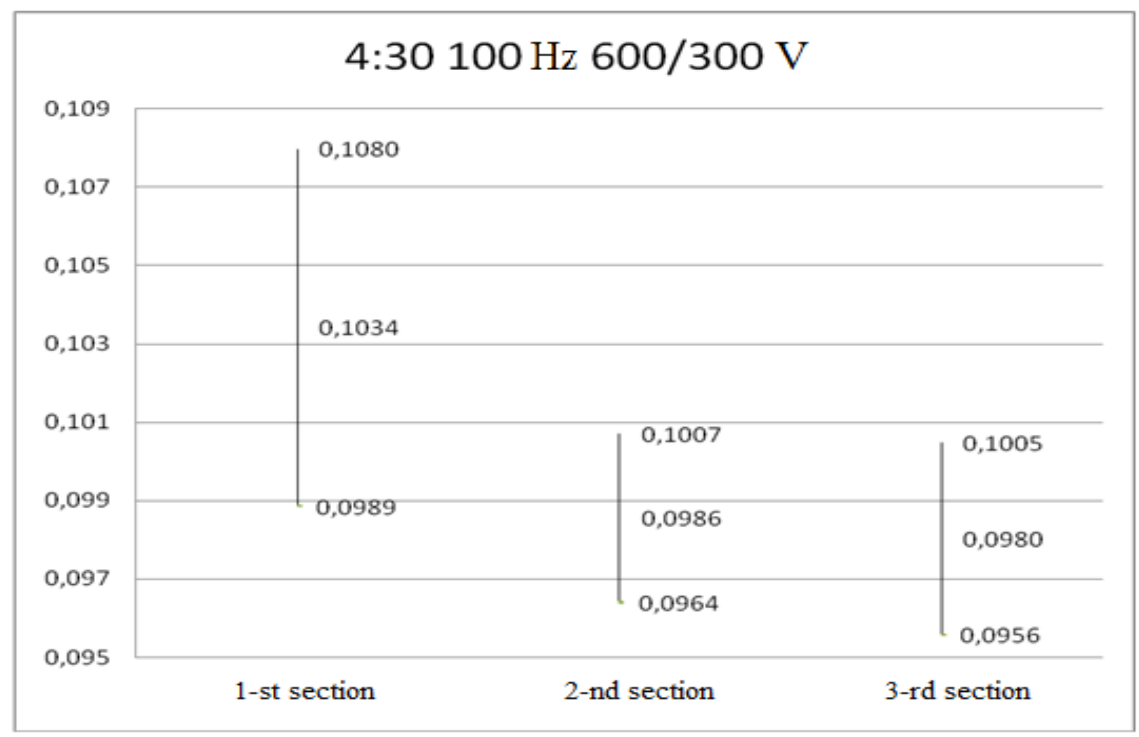

Fig. 4. Cerium cations concentrations (g/l) in experimental cell after field action for 4 hours 30minutes. $100 \mathrm{~Hz}, 600 / 300 \mathrm{~V}$.

The performed experiments prove the possibility to use previously found effect of selective electroinduced drifting of solvated cations in salt solutions when influenced by asymmetric electric field for organizing production method of solutions enrichment by the target metal. The experimental results proved previously obtained theoretical suppositions according to which at low salt concentrations (about $0.1 \mathrm{~g} / \mathrm{l}$ ) the solvated ions drifting effect induced by external electric field is excited in aqueous solution at relatively low frequencies. It remains to suppose that solvated ions motion excited by the action of external asymmetric electric field is more complex, or that the clusters are formed by several ions, and not by the only ion and associated solvent molecules.

\section{EXPERIMENTAL RESULTS (CIRCULATION OF SOLUTION)}

Selective drift (directed motion) of cationic aquacomplexes in solutions under external asymmetric electric field action was studied in experimental separation cell construction of which is shown on Fig. 5.

The cell was loaded with solution of mix of salts $\mathrm{CaCi}_{2}$ and $\mathrm{MgCl}_{2}$ in water. Concentration of salts was $2 \mathrm{~g} / \mathrm{l}$. The structure of samples of solution was defined by means of the X-ray exited fluorescent radiation analysis. Thus the characteristic spectrum was excited by the brake radiation of the chromic anode and was registered by planar semiconductor Si:Li detector. Generation of brake radiation was in a mode of the X-ray tube: $20 \mathrm{kV}, 100 \mu \mathrm{A}$. 

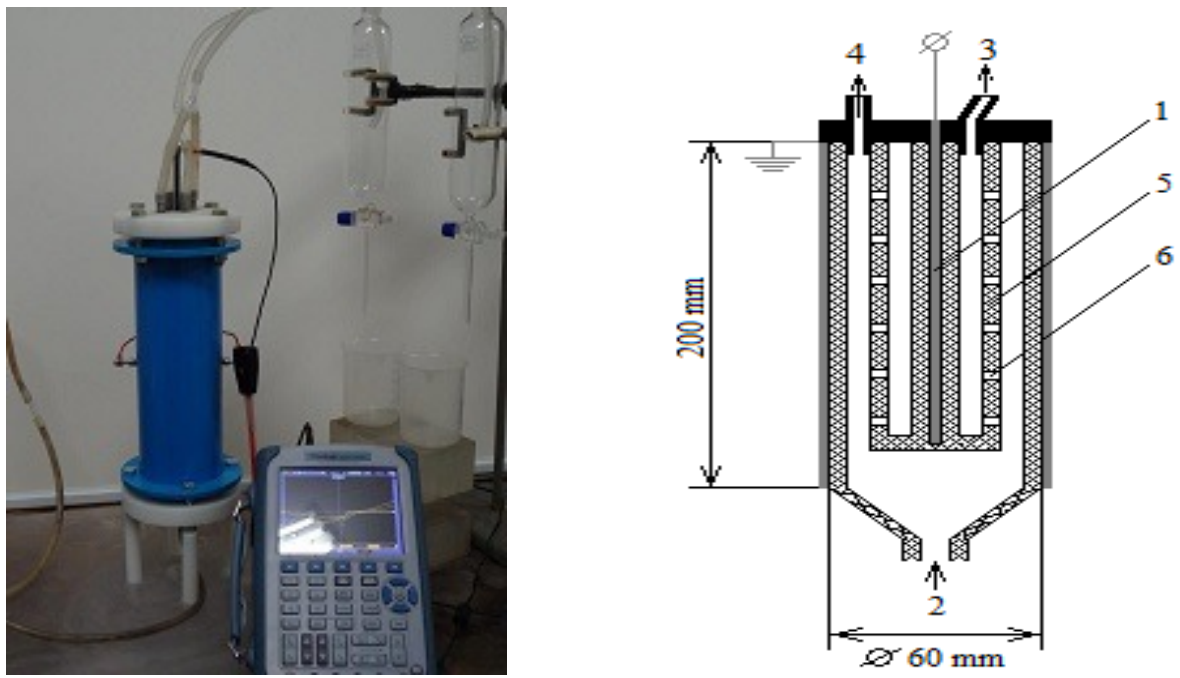

Fig. 5. Separation cell: 1 - potential electrode, 2 - input of solution, 3 - output of enriched solution, 4 - output of depleted solution, 5 - internal cylinder for separation of solution flow, 6 - openings (all the elements are made from insulating materials)

Potential of one of electrodes of separation cell changed in time as sine, but with different amplitudes in half-cycles $U(t) \approx A(1+2 \sin \omega t-\sin 2 \omega t)$. Speed of pumping of solution through a cell was $7 \mathrm{l} / \mathrm{h}$.

On Fig. 6 the spectrum of fluorescent radiation of the sample of solution is shown as an example.

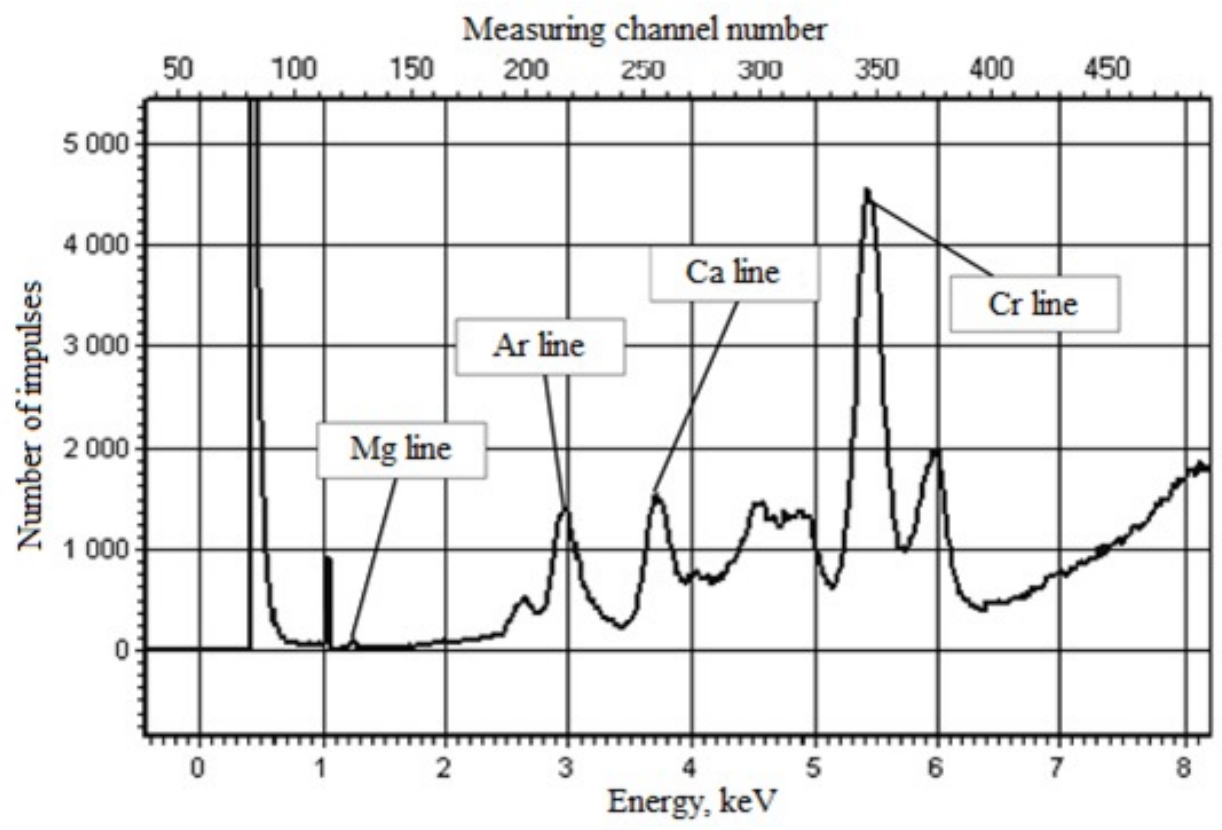

Fig. 6. Example of reading of spectrum by means of Software SPECTR7

Intensity of the line of chemical element is proportional to its concentration. Intensity of the $\mathrm{Ca}$ and $\mathrm{Mg}$ lines were always normalized on intensity of the Ar line. It is thus supposed that the content of argon in air during all measurements is constant $(0.93 \%)$. 
On Fig. 7 the spectrum of radiation of sample of solution at input of the separation cell (position 2 in Fig. 5) is shown.

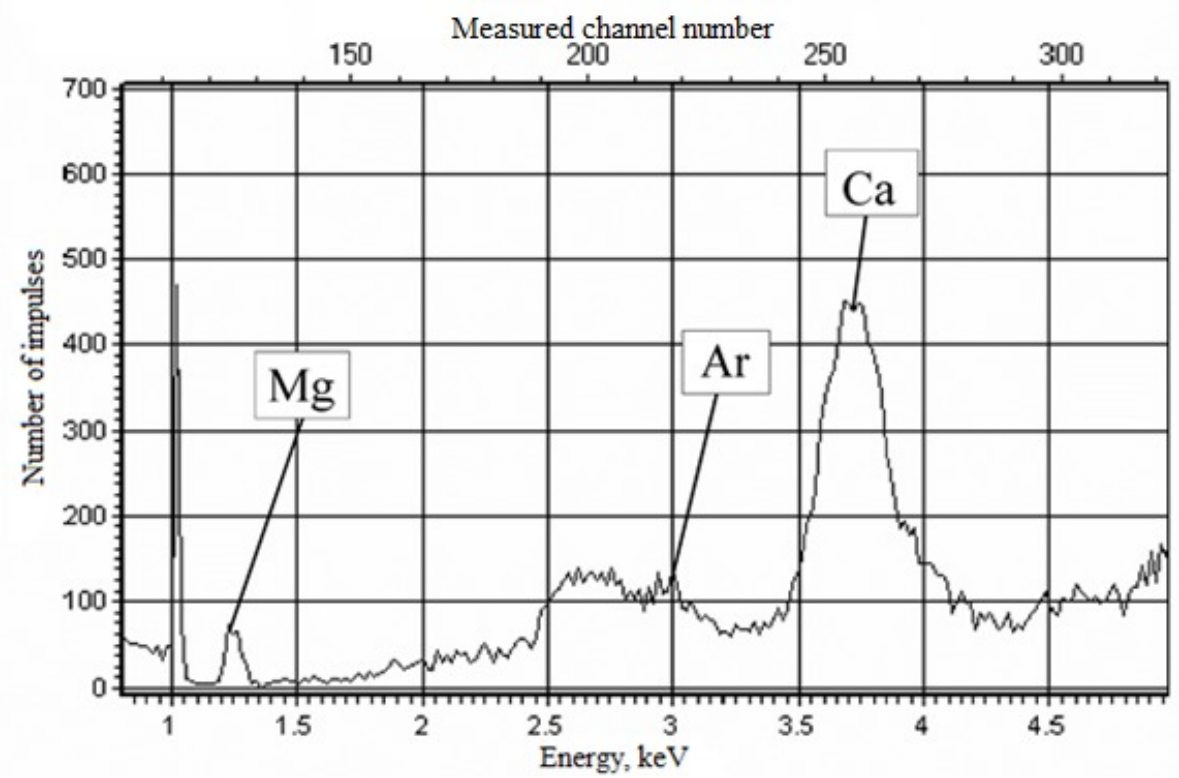

Fig. 7. The spectrum of sample of feeding solution

On Fig. 8 the spectrum of radiation of sample of solution which is enriched with $\mathrm{Ca}$ ions at output of the separation cell (position 3 in Fig. 5) is shown.

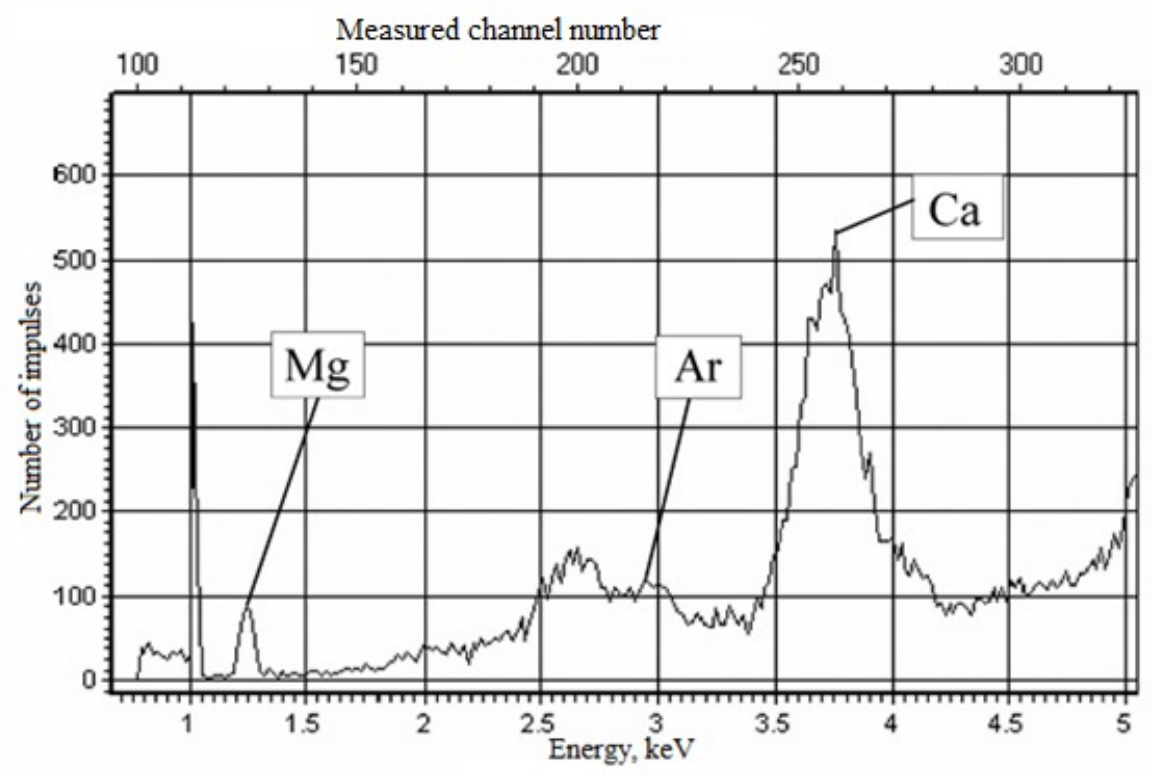

Fig. 8. The spectrum of radiation of sample of solution which is enriched with $\mathrm{Ca}$ ions

On Fig. 9 the spectrum of radiation of sample of solution which is depleted in Ca ions at output of the separation cell (position 4 in Fig. 5) is shown. 


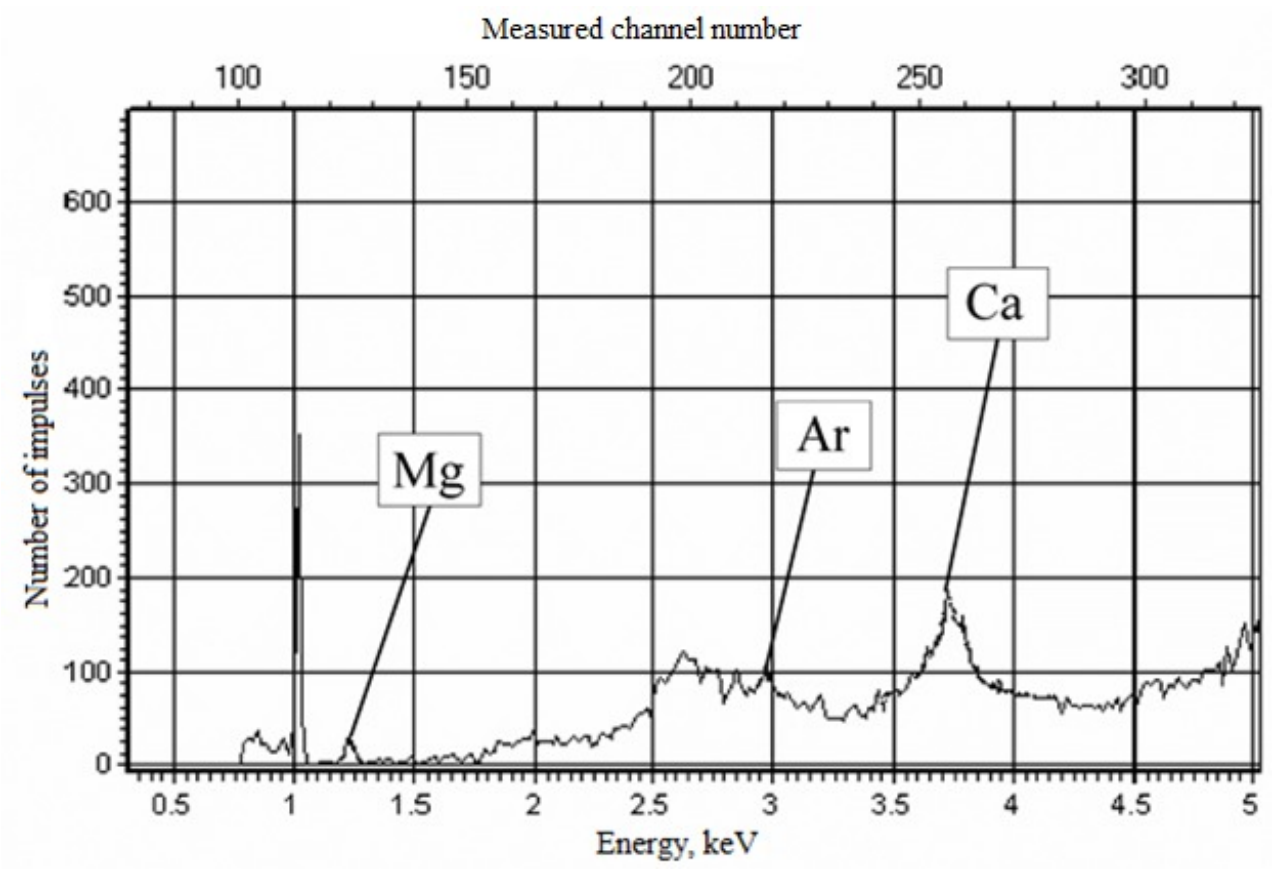

Fig. 9. The spectrum of radiation of sample of solution which is depleted in $\mathrm{Ca}$ ions

\section{DISCUSSION}

Changes of concentration of ions in solution which are shown on Figs. 5 and 6 happened when the frequency of potential $\mathrm{U}$ was $80 \ldots 100 \mathrm{~Hz}$ and amplitude of potential A was $80 \mathrm{~V}$.

Thus the effect of separation of the solvated ions is exciting when the frequency of external electric field is $\sim 100 \mathrm{~Hz}$. As this frequency has to correlate with natural frequency of the solvated cations, the sizes of the solvated ions (clusters) has to be $\sim 0.1 \mu \mathrm{m}$. At such sizes the moment of inertia of a cluster corresponds to frequency $\sim 100 \mathrm{~Hz}$.

The process of ions salvation in solutions of salts in liquid polar dielectrics provides an opportunity of development of essentially new technologies and techniques. The conditions providing use of mass transfer process for the resolution of various problems of application, first of all are defined by the sizes of solvated ions (clusters). In particular, action of periodic electric fields with various combinations of frequency, strength and the dependence of amplitudes of strength in half-cycles on solutions of salts in polar dielectrics causes excitation of rotary-forward motion solvated ions (clusters) and, hence, mass transfer [4]. The frequency of these fields does not exceed a few of kilohertz and the amplitude of field strength in solutions not exceeds tens of volts on centimeter. Thus electrodes, by means of which in volume of solution various configurations of an electric field are created, are isolated from solutions. Experimental data and theoretical estimations show, that distinction of inertial properties of the solvated ions, caused in the different sizes of the solvated shell, can be necessary in a basis of technology of element enrichment of solutions of salts of metals. It is possible to expect, that it will find utilizations also in other effects exiting by the influence of electric and magnetic fields on solutions of salts.

\section{CONCLUSION}

At present time the mechanism of ion-molecular interaction in solutions is not clear. There are experimental data which testify that the size of solvated ions covers in water electrolytes is equal to several tens of sizes of water molecules. There are also experimental data which testify that placing of volume distributed electric charge in dielectric liquid is accompanied by formation of the supramolecular particles linear sizes of which reach value about $1 \mu \mathrm{m}$. 
At determination of amplitude-frequency parameters of electric fields by means of which it is possible to excite an ionselective mass transfer in solution, we needed correct determination of the sizes of covers which are formed from solvent molecules around ions. Thus the traditional description of solvated ions properties based on Debye-Huckel approach, led us to that the ion-selective mass transfer in solution has to take place with frequencies of external electric field $\sim 10$ MHz. Nevertheless, really ion-selective mass transfer in solution was excited with frequencies $\sim 100 \mathrm{~Hz}$.

Therefore the problem of the description of process of orientation and holding of dielectric solvent molecules in the field of ion was posed and solved. Results of the solution of this problem testify that placing of volume distributed electric charge of ion in dielectric liquid is accompanied by formation of the supramolecular particles, which we called "clusters", linear sizes of which is significantly more than first and second radiuses of solvation ( 1 Angstrom) and reach size $\sim 0.1$ microns. At such sizes inertial properties of clusters and their natural frequencies give the chance to operate their movement by means of action of external electric field on solution.

The conducted theoretical assessment point out the possibility of associates (clusters) formation from solvated ions in salt solutions in dielectric liquids. It is probable that the action of external periodic electric field with different intensity amplitudes in half-periods causes directed motion not of separate solvated ions, but associates-clusters formed by groups of solvated ions. Significantly bigger mass of the associate and, consequently, bigger value of the inertia moment explain the manifestation range shift of elecroinduced drifting effect of solvated ions towards smaller frequencies at salt concentrations to $10 \mathrm{~g} / \mathrm{l}$, which is coherent with the experimental results.

\section{ACKNOWLEDGMENTS}

We are grateful to Dr. Lomov I.V. and Dr. Egorov N.B. for carrying out the analysis of chemical composition of samples of the solution. Also we are grateful to academician Trutnev Yu.A. for discussions on physical bases of observed effect.

\section{REFERENCES}

[1] Kazaryan M. A., Lomov I. V., Shamanin I. V., [Electro-physics of structured solutions of salts in liquid polar dielectrics], Moscow: Fizmatlit, (2011).

[2] Ebert G., [Short handbook on physics], Moscow: Fizmatgiz, (1963).

[3] Nevolin V. K., "Physical principles of scanning-tunnelling nanotechnology," Electronic industry 10, 8-15 (1993).

[4] Shamanin I. V., Kazaryan M. A., "The use of electrical induced selective drift of solvated ions in solutions phenomena in technologies," International Journal of Research in Physical Chemistry 2(4), 40-44 (2012). 